\title{
Espaces protégés, espaces sanctuarisés ? Conflits autour de la loi littoral en Corse
}

Protected area or sanctuary? Conflicts over the legal protection of coast in Corsica

\section{Romain Melot et Jean-Christophe Paoli}

\section{OpenEdition}

\section{Journals}

Édition électronique

URL : http://journals.openedition.org/economierurale/3661

DOI : 10.4000/economierurale.3661

ISSN : 2105-2581

\section{Éditeur}

Société Française d'Économie Rurale (SFER)

Édition imprimée

Date de publication : 30 novembre 2012

Pagination : 60-73

ISSN : 0013-0559

Référence électronique

Romain Melot et Jean-Christophe Paoli, «Espaces protégés, espaces sanctuarisés ? Conflits autour de la loi littoral en Corse », Économie rurale [En ligne], 332 | novembre-décembre 2012, mis en ligne le 30 décembre 2014, consulté le 20 avril 2019. URL : http://journals.openedition.org/ economierurale/3661 ; DOI : 10.4000/economierurale.3661 


\section{Espaces protégés, espaces sanctuarisés ? Conflits autour de la loi littoral en Corse}

Romain MELOT ? INRA SADAPT, Paris

romain.melot@agroparistech.fr

Jean-Christophe PAOLI ? INRA LRDE, Corte

paoli@corte.inra.fr

\section{Introduction}

$\grave{A}$ la suite des réformes de décentralisation, la loi littoral (et son alter ego, la loi montagne) a été conçue comme le pendant des pouvoirs importants octroyés aux communes en matière d'urbanisme et de planification : il s'agissait de limiter l'étalement urbain et l'appétit des promoteurs dans des zones où les espaces agricoles et naturels étaient soumis à une forte pression. Si l'on a pu estimer que dans de nombreux cas, cette loi est arrivée « trop tard » (urbanisation de la Côte d'Azur), les conditions de son application se sont en revanche rapidement posées avec une particulière acuité dans les régions où d'importantes réserves foncières demeuraient disponibles, du fait de la relative préservation des espaces côtiers.

Soucieux de développer, les infrastructures touristiques, les élus des communes littorales corses se sont peu à peu mobilisés pour demander l'assouplissement de la loi, idée relayée au niveau de l'exécutif régional par le projet, maintes fois avorté, de redéfinir localement les dispositions légales dans le cadre d'un plan d'aménagement de l'île. Dans le même temps, la question de la protection du littoral est devenue pour les services de l'État un enjeu d'affirmation de l'ordre public notamment à partir de la fin des années quatre-vingt-dix. La mise en conformité des documents d'urbanisme communaux avec la loi littoral focalise alors les crispations entre élus locaux et représentants de l'administration déconcentrée. De nombreuses décisions des élus se retrouvent déférées par le préfet devant le tribunal administratif, signe que les mécanismes de négociation habituels (accommodements concernant les règlements d'urbanisme définis par les communes) ne s'avèrent plus en l'état de fonctionner.

Ces évolutions institutionnelles interviennent alors que les espaces non bâtis du littoral se trouvent dans une situation de fragilité caractérisée par une double concurrence avec les intérêts de l'économie touristique et résidentielle. Tandis que les espaces naturels les plus fragiles correspondent aux zones d'implantation touristique les plus recherchées, l'agriculture et l'élevage tendent à se concentrer le long de plaines côtières irriguées. Cette évolution est due à l'abandon des cultures en sec des arrièrepays et à l'intensification. Par voie de conséquence, les zones rurales et naturelles préservées proches du rivage se retrouvent dans une situation de rareté et d'intense pression foncière.

L'objet de cette étude vise à mettre en évidence les logiques qui président à l'usage de la justice administrative comme lieu de traitement des conflits relatifs aux usages du littoral. Nous faisons en effet l'hypothèse que l'étude de la conflictualité et de son traitement judiciaire en particulier permet de mieux appréhender les types d'intérêts et d'usages qui soutiennent la demande de constructibilité (Torre, Caron, 2005). Cette perspective empirique vise également à identifier les types de décisions les plus fortement visées par les controverses. Dans cette perspective, nous nous intéresserons à l'usage de la loi littoral comme référent parmi d'autres, mobilisé devant les juges pour la préservation des espaces non bâtis dans des zones à forte pression foncière. 
Les dispositions de la loi littoral constituent-elles un référent efficace là où d'autres dispositifs, comme les règlements des documents d'urbanisme, se révèlent insuffisants pour limiter l'urbanisation?

Nous nous appuierons sur l'analyse de l'ensemble des affaires portées devant le tribunal administratif de Bastia durant les années 2004 à 2008 (soit un peu moins de deux cents affaires) ayant pour objet le respect de ces dispositions de la loi littoral (contestations relatives aux permis de construire et aux documents d'urbanisme) en articulant l'analyse autour de trois catégories de données : le profil des acteurs des procès, la typologie des décisions objets de litige, ainsi que les registres d'argumentation développés par chacun auprès du tribunal ${ }^{1}$. Après avoir présenté le contexte dans lequel s'exerce la pression foncière sur les espaces littoraux de l'île (1), nous examinerons le rôle stratégique joué par les services de l'État à la fois comme autorité délivrant des autorisations et comme acteur clé du contentieux (2). Enfin, nous prolongerons notre propos par une analyse plus approfondie des registres d'argumentation mobilisés par les auteurs des recours devant la justice administrative (3).

\section{Un espace convoité Le contexte de la pression foncière sur les espaces littoraux}

\section{Un littoral longtemps délaissé}

Les appétits et controverses qui entourent le littoral corse ne laissent pas de surprendre lorsque l'on mesure à quel point de larges parties du littoral corse restent vides d'habitation. En effet, la majorité de l'espace

1. L'échantillon de jugements étudié a été constitué via l'interrogation du Fonds d'archives des tribunaux administratifs, qui recense de manière exhaustive les jugements statuant sur le fond. Sur la période retenue pour l'étude (les quatre dernières années, soit les jugements prononcés de 2004 à 2008), l'échantillon est constitué de 187 jugements concernant uniquement le contentieux lié à la loi littoral. littoral demeure relativement peu bâti, que ce soit sur la côte ouest, rocheuse et montagneuse ou sur la côte est, plane et sablonneuse. Historiquement, l'espace littoral corse est marqué par une urbanisation peu dense, mais maillée d'activités humaines rurales qui ont imprimé un bâti rural dispersé composé de maisons rurales, bergeries et autres bâtiments ruraux anciens parfois regroupés en hameaux. C'est dire que les espaces littoraux corses ont une particularité historique - qui se retrouve en partie dans la Sardaigne voisine - caractérisée par l'éloignement des villages vers l'intérieur d'une part, l'habitat dispersé de plaine d'autre part (Paoli, 2000).

L'ancien système agro-pastoral à transhumance inverse, décrit par le géographe Raoul Blanchard, était basé sur un centre habité situé à mi-pente (souvent entre quatre et sept cents mètres d'altitude) où se concentraient les cultures permanentes et intensives (Blanchard, 1914). Le bétail élevé sur parcours, ne pouvant pas subsister à ces altitudes en hiver, « descendait » d'octobre à mai vers des zones de basse altitude, lesquelles sont en Corse en général proches du littoral. Dans ces zones, les paysansbergers pratiquaient des cultures céréalières sur brûlis, nécessaires à la fois pour la provision des céréales et l'entretien des parcours. Beaucoup de villages de haute altitude (plus de sept cents mètres) étaient d'ailleurs entièrement dépendants pour leur pain de ces emblavures lointaines. C'était alors la totalité de la population qui jusqu'à la veille du $\mathrm{Xx}^{\mathrm{e}}$ siècle, descendait en hiver en un mouvement de véritable semi-nomadisme vers les zones côtières, où les familles s'installaient dans des habitations provisoires dispersées pour se regrouper à nouveau vers les villages au printemps suivant.

Bon nombre de communes littorales actuelles ne sont ainsi que les émanations très récentes (parfois un siècle à peine) de ces villages de montagne. Porto Vecchio, troisième ville de l'île avec dix mille habi- 
tants, est « issue » de Quenza (deux cent quarante habitants). Il va sans dire qu'entre la création de ces communes et le développement touristique littoral la prévalence économique entre ces deux ensembles (village de montagne d'un côté, littoral et arrière pays côtier - appelé «plage » (piaghje) par les Corses - de l'autre) s'est complètement inversée. Le développement de la mécanisation agricole, des activités tertiaires autour des villes et finalement du tourisme ont donné tout au long du $\mathrm{XIX}^{\mathrm{e}}$ siècle la prééminence aux «plages » alors que les villages se vidaient jusqu'à peu de leur population. Cette prééminence économique se retrouve dans le tracé des communes puisque, là où les divisions communales n'ont pas eu lieu, les agglomérations touristiques importantes restent dépendantes de villages souvent bien moins opulents situés dans les montagnes.

À la différence des situations provençales ou liguriennes, le littoral corse n'est pas structuré par une trame d'urbanisation continue autour d'agglomérations importantes, susceptible de favoriser une généralisation du mitage. Cette pression urbaine opérée par une spéculation ancienne est à l'origine de stratégies qui ont pu être mises en évidence sur le littoral varois ; stratégies visant à profiter de multiplier les autorisations d'urbanisme dans des secteurs souséquipés, en jouant des possibilités offertes par le droit des documents d'urbanisme (Daligaux, 1996).

À l'inverse, les communes littorales corses ne se sont dotées souvent que tardivement de documents d'urbanisme, l'urbanisation touristique se concentrant jusqu'aux années quatre-vingt autour de sites ciblés. En revanche, à l'instar des espaces méditerranéens continentaux, le territoire des communes côtières de l'île obéit à une structuration opposant une partie littorale sur laquelle se concentrent les enjeux et une partie intérieure et montagneuse, généralement peu concernée par la spéculation foncière. Les élus sont d'autant plus enclins à protéger ces territoires de l'arrière-pays, que ces espaces, boisés et enclavés, sont peu convoités par les promoteurs. Ils présentent en outre l'avantage indéniable de pouvoir être affichés pour promouvoir l'image d'une commune respectueuse de ses obligations de préservation, suivant un mécanisme de justification juridique et politique déjà identifiés sur d'autres espaces littoraux (Daligaux, 2003).

\section{L'enjeu économique de l'urbanisation littorale}

Figure 1. Surfaces autorisées annuellement (en $\mathrm{m}^{2}$ ) au titre des permis de construire dans les communes littorales corses (moyenne sur la période 1997-2007)

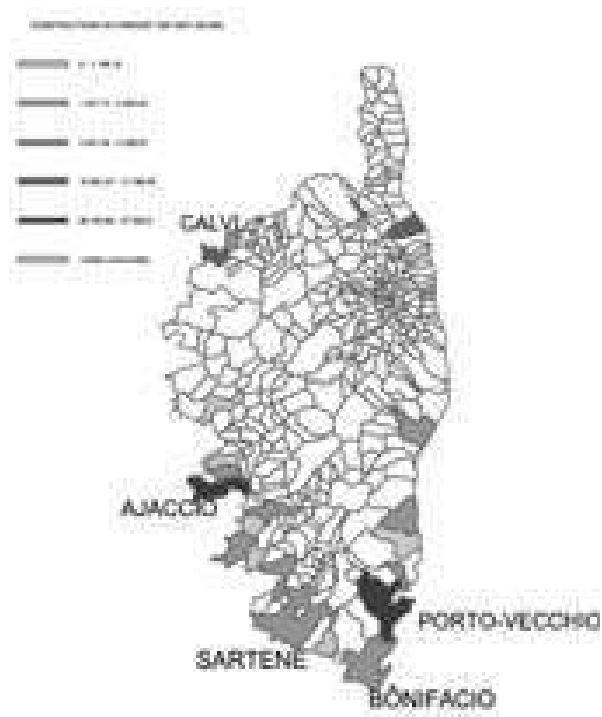

Source : carte réalisée d'après les données de la base Sitadel, MEDAD

Comme le montre la carte des surfaces autorisées au titre des permis de construire sur le littoral corse (figure 1), le cœur du marché de la construction (en plus des deux agglomérations d'Ajaccio et de Bastia) se situe sur le littoral, en particulier dans la partie sud de l'île (Porto-Vecchio, Bonifacio, Sartène, Propriano figurant parmi les communes les plus concernées). À cela s'ajoute que le dynamisme immobilier du sud de l'île se traduit par une valeur moyenne des terrains 
supérieure à celle pratiquée dans la partie nord $^{2}$. On comprend de ce fait que les élus locaux du sud de l'île aient été les plus actifs avocats d'un assouplissement de la loi littoral lors de la procédure d'élaboration du plan d'aménagement régional au vu des intérêts en jeu ${ }^{3}$. La protection du littoral par la législation d'urbanisme est souvent présentée par ces responsables politiques comme une entrave au développement de l'île et une source de contraintes incompatibles avec le plein exercice des compétences issues de la décentralisation. L'examen des données disponibles montre cependant que la période qui a suivi la mise en place de la loi littoral a coïncidé avec une forte croissance du marché de la construction.

En effet, contrairement au discours souvent relayé par les élus des communes littorales touristiques, la période qui a suivi la mise en place de la loi littoral se traduit par une augmentation quasi-continue des autorisations d'urbanisme sur le littoral. Les données du marché de la construction concernant le département de la Corse-duSud - le plus stratégique, comme nous l'avons vu - indiquent que les surfaces autorisées au titre des permis de construire dans les communes littorales durant la période allant de 1990 à 2007 ont augmenté

2. Le prix moyen des terrains à bâtir en Corse-duSud (toutes surfaces confondues) était en 2009 de 100300 euros (à peu près la moyenne enregistrée dans les départements du Gard et du Vaucluse), soit une valeur moyenne supérieure d'un tiers à celle observée dans le département de Haute-Corse (66 900). Source : base de données Perval (données publiques agrégées).

3. Serpent de mer de la politique locale en Corse, l'idée d'un document d'urbanisme régional élaboré par les élus - ayant entre autres pour objet de préciser les modalités d'application de la loi littoral a été maintes fois reportée, d'abord à la fin des années quatre-vingt puis plus récemment en juillet 2009 par le report du débat en Assemblée territoriale sur le Plan d'aménagement et de développement durable de la Corse. Voir à ce propos Paoli J.-C., Melot R., Fiori A. (2008). de manière continue et importante, les projets de constructions individuelles isolées ou groupées évoluant d'ailleurs de manière similaire (la baisse des autorisations de bâtiments collectifs sur la période ne modifiant pas de manière significative cette tendance générale $)^{4}$. Si la loi littoral a pu être perçue par les élus comme une contrainte importante (Celli, Garcia, 2002), elle a sans doute davantage entravé des projets situés à proximité immédiate du rivage qu'elle n'a enrayé la tendance globale à l'urbanisation des zones côtières.

L'enjeu économique de l'urbanisation littorale apparaît nettement au vu de la structure des activités et de l'emploi dans l'île. Deux éléments saillants de l'économie de la Corse sont à souligner de ce point de vue : la place importante du secteur de la construction et le poids du secteur touristique dans l'emploi salarié. Région la moins industrialisée de France, la Corse est marquée par une économie fortement tertiarisée, au sein de laquelle le secteur de la construction pèse plus lourd (9 \%) que le secteur industriel $(7 \%)$ du point de vue de l'emploi salarié (Levratto, 2001). Quant au tourisme, même si l'emploi tertiaire demeure très largement dominé par la fonction publique d'État, sa place est prépondérante concernant le tertiaire privé (jusqu'à 6000 salariés durant la saison estivale). À cet égard, la Corse est représentative de la forte spécialisation des «petites économies insulaires » en faveur de la production de services pour les particuliers, essentiellement dans le tourisme et le commerce. (Maupertuis, Logossah, 2007).

4. À l'exception des années 1994, 1996 et 2004, le marché de la construction est caractérisé par une croissance très régulière sur la période de 1991 à 2007, passant de $60000 \mathrm{~m}^{2}$ autorisés environ en 1991 à près de $100000 \mathrm{~m}^{2}$ autorisés en 2007 (source : base SITADEL). 


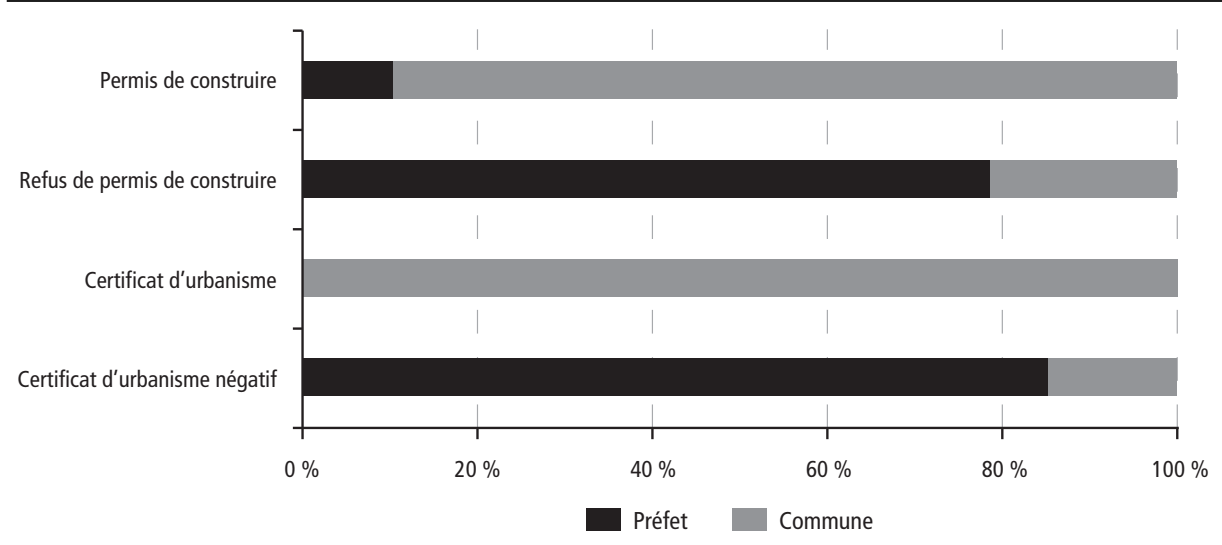

Source : Affaires Trib. Admin., Bastia, 2004-2008

\section{Les services de l'État Partie prenante du contentieux}

\section{Le préfet, autorité de décision et auteur de recours}

Depuis les premières lois de décentralisation, les communes disposent d'une large compétence en matière d'urbanisme à condition d'avoir opté pour une démarche de planification globale, matérialisée par un document d'urbanisme. En l'absence d'un tel document, le préfet demeure seul habilité à délivrer les autorisations individuelles d'occupation du sol, qu'il s'agisse de permis de construire ou de certificats d'urbanisme (attestant de la constructibilité du terrain en l'absence de projet encore défini). Territoire constitué essentiellement de communes rurales et montagnardes, la Corse reste relativement peu dotée en documents d'urbanisme ${ }^{5}$. Si la plupart des zones littorales ont peu à peu rattrapé leur retard en la matière

5. Au 1er janvier 2009, 46,7\% des 360 communes corses étaient dépourvues de document d'urbanisme, contre $32,4 \%$ des communes sur l'ensemble du territoire national. Même en ajoutant aux communes dotées de documents d'urbanisme les communes dont le document en est seulement au stade du projet, la proportion de communes concernées n'atteint que 53,3\% du total, contre $67,6 \%$ sur la France entière (Source : DIACT, Observatoire des territoires). (parfois dû à l'annulation par le tribunal administratif de documents non conformes à la loi littoral) et sont actuellement ou seront à brève échéance couvertes par un document d'urbanisme, d'autres restent tributaires de cet héritage et sont dépourvues de plan local d'urbanisme, de plan d'occupation des sols ou de carte communale. Cet état de fait explique que les décisions dont l'annulation est requise devant le tribunal administratif soient aussi bien des autorisations délivrées par le maire que par le préfet ${ }^{6}$.

La distinction que nous opérons entre autorisations communales et autorisations préfectorales revêt toute son importance lorsque l'on prend en compte le préjudice ou le bénéfice que ces décisions génèrent pour le pétitionnaire, c'est-à-dire la personne (généralement le propriétaire, plus rarement le responsable des travaux) ayant sollicité l'autorisation administrative. Les décisions attaquées qui restreignent l'urbanisation (refus de permis de construire, certificats

6. Un nombre réduit d'affaires (19) de notre échantillon est constitué de recours en annulation de documents d'urbanisme que nous n'avons pas intégrés à l'analyse statistique approfondie en raison de leur caractère particulier : ils sont tous dirigés contre une seule décision, la délibération du conseil municipal de Bonifacio approuvant le plan local d'urbanisme en 2005. 
d'urbanisme négatifs) émanent en effet en grande majorité du préfet. À l'inverse, lorsque les maires sont assignés en justice, c'est dans la plupart des cas concernant des décisions favorables à l'extension du bâti (permis de construire accordés et certificats d'urbanisme positifs). Le contentieux de l'urbanisme littoral revêt donc en Corse une structure clairement binaire, puisqu'il se traduit soit par des recours de propriétaires insatisfaits contre des refus d'autorisations émanant quasi-exclusivement des services de l'État, soit par des recours de tiers contre des décisions des maires répondant le plus souvent favorablement aux demandes du pétitionnaire.

Deux enseignements peuvent être tirés de ces premières observations. Tout d'abord, le fait que les refus d'autorisations émanent quasi-exclusivement du préfet semble montrer que les élus sont réticents à résister aux demandes de leurs administrés en matière de constructibilité. Si ces raisons sont aisément explicables (pression électorale, volonté de faciliter la réalisation de la rente foncière et de promouvoir l'activité touristique), la proportion de décisions de refus apparaît cependant particulièrement faible. Les maires des communes littorales semblent donc peu disposés à assumer leur fonction de « filtrage » des demandes en fonction de l'impact environnemental qu'elles sont susceptibles de causer. Et ce d'autant plus que les demandes n'émanent pas que de particuliers, mais également de personnes morales porteuses de projets de plus grande ampleur ${ }^{7}$. Cette sensibilité des élus aux intérêts économiques concernant l'usage du littoral est d'ailleurs parfaitement décrite par des enquêtes réalisées dans l'ouest de la France sur le contentieux pénal de l'urbanisme : alors que les associations de

7. Environ un pétitionnaire sur cinq présent dans les affaires (au titre de demandeur ou de défendeur) est une personne morale (sociétés civiles immobilières, sociétés commerciales) et un sur dix une société commerciale. protection de l'environnement peinent à faire aboutir les poursuites, les élus soutiennent fortement les plaintes du secteur hôtelier contre les activités susceptibles de leur porter préjudice - camping-caravaning, mobilhome... (Struillou, 2004).

\section{Le rôle joué par les tiers dans les recours contentieux}

Face à cette interrogation, on peut certes considérer les recours contentieux initiés par les tiers comme un instrument efficace de régulation : ils contribuent à consolider l'application des règles protectrices de la loi littoral en soumettant les autorisations litigieuses au contrôle du juge administratif (Lascoumes, 1995). Cependant, l'examen de la situation observée dans le cas du littoral corse ne peut qu'aboutir à nuancer ce propos, et ce pour deux raisons. Les recours « nimbyistes » initiés par les particuliers riverains des projets de construction demeurent tout d'abord relativement peu nombreux, tandis que les associations même si certaines de leurs actions en justice ont bénéficié d'une importante médiatisation - représentent une part encore moindre de ces procédures. Au total, les recours initiés par ces tiers ne constituent que $16 \%$ des affaires (dont $7 \%$ de recours associatifs) et aboutissent à des résultats mitigés (l'annulation de la décision n'est obtenue que dans un cas sur deux).

La difficulté des associations aux moyens parfois limités à investir le terrain du prétoire sur plusieurs fronts à la fois et le choix de d'autres modes d'intervention (pressions directes auprès des élus, médiatisation) peuvent expliquer cet activisme judiciaire limité. Au total, les recours initiés par ces tiers ne constituent que $16 \%$ des affaires (dont $7 \%$ de recours associatifs) et aboutissent à des résultats mitigés (l'annulation de la décision n'est obtenue que dans un cas sur deux). Peu présentes sur le plan de la fréquence des recours, les associations ont en revanche un taux de réussite devant le tribunal supérieur à celui des 
autres demandeurs. Ce phénomène peut s'expliquer par leur aspect sélectif. Le profil de ces associations est double : il s'agit soit de groupements dont l'action est à la fois ciblée thématiquement et géographiquement (comme $\mathrm{ABCDE}$ auteur de plusieurs recours contre le PLU de Bonifacio ou l'Association pour le libre accès aux plages à Porto-Vecchio), soit d'associations ayant eu à l'origine un ancrage local, mais dont la sphère d'action s'est peu à peu élargie au gré des engagements. Elles conjuguent alors généralement différents outils d'intervention (expertises, campagnes médiatiques, revendications auprès des élus), dont l'arme contentieuse n'est qu'un élément. Élément qui, par défaut, peut s'imposer comme moyen d'action ultime lorsque l'administration où les élus les écartent des procédures de concertation en raison de leur caractère contestataire. L'association U Levante illustre bien ce second profil, puisque née d'une contestation contre l'urbanisation d'un site classé, elle a étendu ses statuts à la préservation de l'environnement en général et sur l'ensemble du territoire de l'île. Évincée de la phase de concertation préalable à l'élaboration du plan régional d'aménagement, elle a mobilisé avec succès le registre médiatique et judiciaire.

Si les recours des tiers constituent en définitive une part importante des affaires traitées par le tribunal (pratiquement la moitié), c'est moins en raison de l'initiative des riverains et des associations que de l'activisme judiciaire de l'administration : près d'une affaire sur trois prend la forme d'un déféré préfectoral, c'est-à-dire un recours contentieux à l'initiative du préfet. À cet égard, le représentant de l'État correspond, plus encore que les associations environnementalistes, à la figure de « l'usager régulier » du tribunal, telle que définie par les sociologues du droit par opposition à l'usager occasionnel qu'est le particulier riverain d'un projet : son action se situe dans une stratégie à long terme et non dans l'expression immédiate d'un préjudice (Galanter, 1974). Lorsqu'elle utilise l'arme judiciaire, l'administration le fait en outre avec un succès important, puisqu'elle obtient l'annulation de la décision litigieuse dans un peu plus des trois-quarts des affaires $(77,8 \%)$, proportion exactement inverse chez les pétitionnaires $(24 \%)$ qui peinent à convaincre les juges. Les services de l'État jouent donc à double titre un rôle central dans la régulation des autorisations d'urbanisme : d'une part, en tant qu'autorité délivrant elle-même des autorisations susceptibles d'être attaquées, et d'autre part, comme usager du tribunal requérant l'annulation d'autorisations délivrées par les maires.

Cependant, ces résultats donnent sans doute une image excessive de l'intensité du contrôle exercé par l'administration sur la légalité des autorisations d'urbanisme au regard de la loi littoral. La prise en compte de la répartition géographique du contentieux telle que cartographiée ci-dessous (figure 3) est à cet égard riche d'enseignements. Si un grand nombre de communes littorales est concerné par ce contentieux (un peu moins de la moitié des quatre-vingtdix-sept communes littorales corses se retrouve citée dans les affaires), la grande majorité des affaires se concentre dans la zone sud et surtout près de la moitié des affaires se répartit dans deux communes de cette zone, Bonifacio et Porto-Vecchio ${ }^{8}$. La concentration du contentieux dans la zone sud est la conséquence des autres phénomènes de concentration constatés plus haut à propos des constructions autorisées : la fréquence des affaires est le reflet des phénomènes sous-jacents que sont l'intensité du marché de la construction et l'afflux de

\footnotetext{
8. Les communes les plus concernées par les affaires sont respectivement : Bonifacio (39 affaires), PortoVecchio (28), Coti-Chiavari (8), L'Ile-Rousse (7), Serra di Ferro (5), Olmeto (4). Les autres communes sont citées à moins de quatre reprises dans les jugements collectés.
} 
demandes d'autorisation dans les communes de cette zone ${ }^{9}$. À ceci près que le poids particulier occupé par les deux communes citées - sans commune mesure avec les données du marché de la construction, même si elles sont le siège d'un important marché immobilier - s'explique également par des particularités locales de gestion de la pression foncière.

En effet, le paradoxe de ces deux communes est de refléter suivant deux mécanismes différents les conséquences d'un même système politique local, organisé de manière à ne filtrer que très faiblement les demandes d'autorisations de construire sur l'espace littoral. Les deux communes ont en commun de présenter des sites et paysages particulièrement recherchés dans le marché immobilier haut de gamme et de faire l'objet d'importantes protections au titre de la loi littoral (espaces remarquables) et de la législation sur les sites classés. Dans le cas de Bonifacio, dotée d'un document d'urbanisme depuis les années quatre-vingts plusieurs associations locales considèrent que la municipalité a longtemps répondu favorablement aux demandes d'autorisations visant l'espace littoral. La situation est différente pour Porto-Vecchio, commune qui, en dépit de son poids démographique et politique prépondérant (troisième ville de Corse, longtemps gérée par le chef de la majorité conservatrice sur l'île) a fait le choix assumé et paradoxal de ne pas se doter de document d'urbanisme, choix distinct de sa voisine, mais guidé finalement par les mêmes considérations : accéder de la manière la plus large aux demandes d'autorisations sollicitées par les administrés sans le carcan d'un règlement d'urbanisme contraignant.

9. Pour illustrer ce phénomène de concentration du contentieux dans la zone sud, précisons que plus de la moitié des communes littorales de Corse-duSud sont le siège d'un recours dans l'échantillon étudié, contre seulement un tiers des communes de Haute-Corse.
Figure 3. Localisation des affaires d'urbanisme liées à la loi littoral (autorisations d'occupation du sol) par catégorie de demandeur (préfet et pétitionnaires).

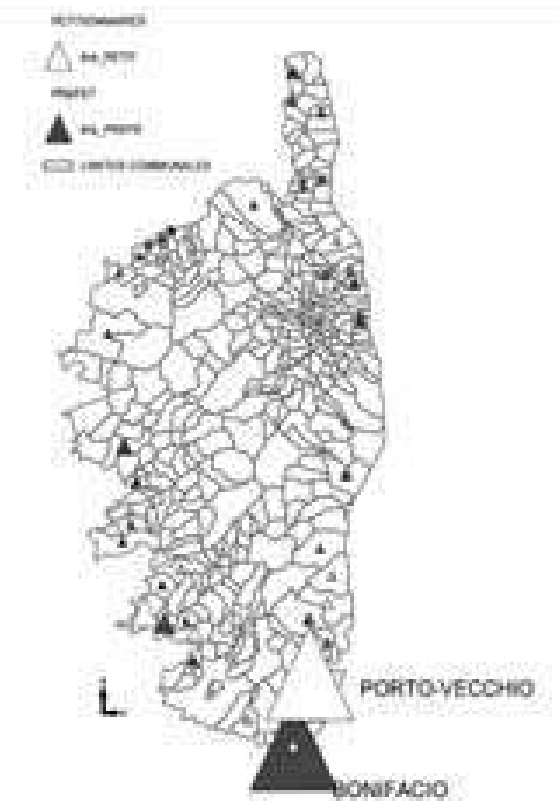

Source : Données de l'enquête

Dans les deux cas, ce système s'appuyait sur la tolérance des services de l'État (acceptant de valider les autorisations sollicitées dans le cas de Porto-Vecchio en l'absence de compétence propre du maire ou s'abstenant de faire usage du déféré administratif pour faire annuler des autorisations illégales dans le cas de Bonifacio). La situation observée sur la période étudiée (2004-2008) est la conséquence d'une politique de réaffirmation de l'autorité de l'État à partir de la fin des années quatre-vingt-dix (notamment à la suite de l'assassinat du préfet Erignac), qui s'est traduite par un durcissement de la position de la préfecture quant au respect de la loi littoral : dans le cas de Bonifacio, multiplication des déférés, et dans le cas de PortoVecchio, des refus d'autorisation. Si le rôle joué par l'administration est donc central dans un grand nombre d'affaires, le caractère fortement localisé des rapports de force politiques qu'il traduit laisse cependant penser que ce rôle risque de se limiter à une conjoncture précise (dans le cas de Bonifacio, par exemple, le conflit entre la 
préfecture et la municipalité peut évoluer à la suite de la défaite de l'ancienne majorité municipale en 2008) et ne présage pas d'un activisme judiciaire de longue durée ${ }^{10}$.

\section{Des registres d'argumentation complexes Le maquis juridique de la protection littoral}

\section{Espaces protégés et enjeux de délimitation}

Les prescriptions issues de la loi littoral définissent des règles d'urbanisation d'autant plus strictes que les espaces concernés sont proches du linéaire côtier ${ }^{11}$. On peut ainsi distinguer, suivant leur degré de sévérité, trois types d'espaces :

- les espaces sanctuarisés : il s'agit d'une part des « espaces remarquables » qui ne tolèrent, en raison de la fragilité et du caractère exceptionnel de leur écosystème, que des aménagements minimaux, et d'autre part, une bande littorale de cent mètres à compter du rivage dans laquelle toute construction est proscrite en l'absence d'urbanisation déjà existante ;

- les espaces où l'urbanisation est réduite : ce sont les « espaces proches du rivage », au sein desquels la loi énonce que l'urbanisation doit être «limitée ». En outre, dans ces espaces, tout projet doit avoir l'aval de la préfecture, même si la commune dispose d'un document d'urbanisme habilitant le maire à délivrer luimême les autorisations ${ }^{12}$;

10. Les trois-quarts des déférés préfectoraux de l'échantillon concernent Bonifacio. Quant aux requêtes visant l'annulation des décisions du préfet, elles concernent à $40 \%$ des affaires situées à PortoVecchio. L'activité du préfet, soit comme auteur des recours, soit comme auteur des décisions attaquées, est donc fortement restreinte géographiquement aux cas de ces deux communes.

11. Codifiées aux articles L. 146-1 et suivants du Code de l'urbanisme

12. Cette obligation d'obtenir l'accord du préfet est cependant levée s'il existe un document d'urbanisme supra-communal définissant des règles de protection auquel le document communal puisse se référer (art. L. 146-4-2). Cette disposition a fortement motivé l'élaboration du plan d'aménagement régional.
- enfin, les espaces où l'urbanisation est canalisée suivant le principe général «d'extension en continuité »: les projets de constructions doivent être conçus dans le prolongement des secteurs bâtis (les « agglomérations et villages existants ») ou bien être suffisamment nombreux pour former une unité urbaine cohérente et de taille limitée (« hameau nouveau intégré à l'environnement »).

Ce sont sur ces différents types de protection que se sont greffées les revendications des élus en faveur d'un desserrement des contraintes. L'importance des enjeux économiques et fonciers sur le littoral est d'ailleurs illustrée au niveau national par l'instabilité de la législation en la matière, objet depuis l'adoption de la loi littoral en 1986 de multiples assouplissements par le biais de « cavaliers législatifs » portés par des élus des zones littorales. Dérogations et exemptions visant, sinon à vider le dispositif protecteur de sa portée - comme c'est pratiquement le cas pour la loi montagne - du moins à restreindre les cas d'application se sont succédées depuis l'adoption de la loi littoral en 1986 - comme les dérogations bénéficiant à certaines installations et à des bâtiments agricoles (Le Cornec, 1997). En outre, les revendications des élus quant à la constructibilité des espaces littoraux peuvent également être émises au nom de l'environnement, en faisant apparaître des conflits d'usage entre lutte contre la pollution et préservation des espaces, comme l'a révélé le débat houleux autour du projet de station d'épuration de Toulon sur le Cap Sicié (Calderaro, 1994).

Dans le cas de la Corse, les débats autour du Plan d'aménagement et de développement durable se focalisent sur la définition de critères de délimitation de ces espaces protégés définis par la loi littoral : le document graphique de la figure 4, extrait de la carte du projet de plan régional, illustrent la manière dont ces délimitations sont opérées. Le zonage des espaces remarquables fait 
Figure 4. Extrait de la carte du projet de Plan d'aménagement et de développement durable de la Corse en 2009 (échelle 1/150 000)

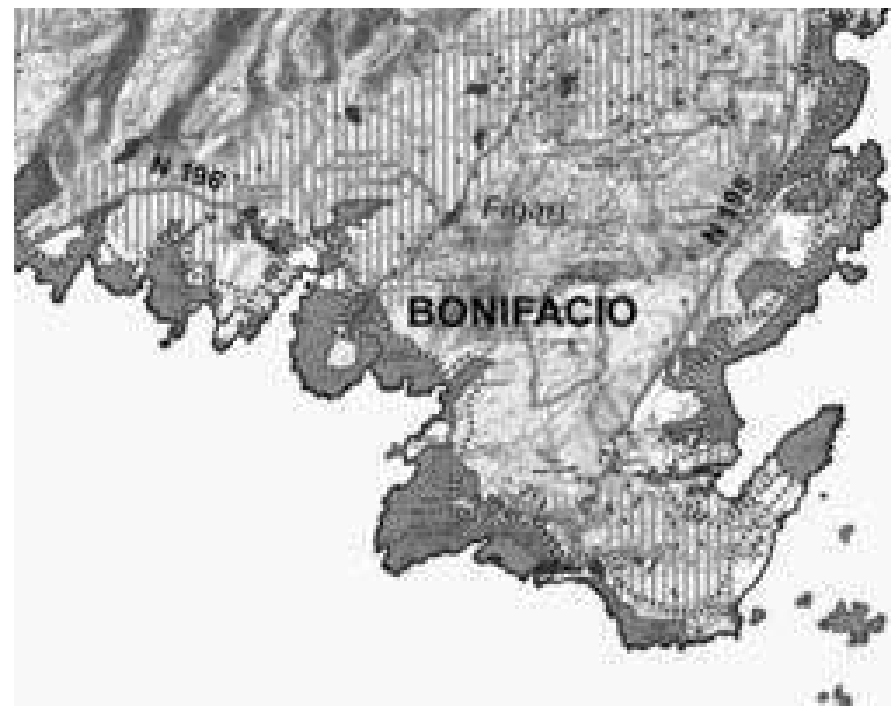

Note : Les espaces remarquables sont indiqués par les zones grisées sur la carte. Les espaces proches du rivage sont délimités par la ligne discontinue.

Source : Association U Levante

l'objet de vives tensions entre élus régionaux et associations environnementalistes en raison de leur valeur patrimoniale. Paradoxalement, les espaces proches du rivage cristallisent encore davantage les controverses: soumis à des protections moins drastiques (l'urbanisation doit y être seulement "limitée") et plus étendus géographiquement, ils correspondent par là même aux zones où les marges de manœuvre pour la constructibilité sont plus étendues. En outre, en l'absence de plan régional, toute décision d'urbanisme prise dans le périmètre de ces espaces nécessite l'accord du préfet. De fait, ce sont sur ce type d'espaces que les demandes des maires sont les plus nombreuses dans le cadre du groupe de travail (Melot, Fiori, Paoli, 2008).

\section{Une typologie de l'argumentation mobilisée devant le tribunal}

Les problèmes d'interprétation de ces dispositions tiennent à plusieurs difficultés logiques. En premier lieu, ces différents types d'espaces ne s'excluent pas les uns des autres, mais peuvent se recouvrir. Ainsi, le principe d'extension en continuité s'applique à l'ensemble du territoire des communes littorales, ce qui inclut également les espaces bénéficiant de protections renforcées $^{13}$. De même, des espaces sanctuarisés comme les espaces remarquables peuvent également être considérés comme « proches du rivage » ou compris dans la « bande littorale », la règle la plus stricte s'appliquant alors. En outre, les différentes catégories juridiques qui définissent ces types d'espaces ont fait l'objet de controverses jurisprudentielles visant à définir des critères de définition des « espaces remarquables » ou des « espaces proches du rivage », ou encore des « espaces urbanisés » et des « hameaux nouveaux ».

13. Le principe général d'extension en continuité de l'existant s'applique également aux communes dépourvues de document d'urbanisme (art. L. 1111-2 du Code de l'urbanisme). Comme les collectivités dans ce cas sont relativement nombreuses en Corse, ce principe est donc mobilisé à double titre dans les affaires (au titre de la loi littoral d'une part, et au titre du «droit commun » de l'urbanisme d'autre part). 
Le principe général d'extension en continuité de l'existant, applicable à l'ensemble du territoire des communes littorales, constitue la règle la plus facilement identifiable et la plus fréquemment mobilisée dans le contentieux. Afin de prévenir le risque de "mitage" des zones littorales, les procédures judiciaires ont amené les juges à établir un certain nombre de critères limitatifs qui traduisent tous une certaine conception d'une urbanisation « acceptable »: le caractère permanent des résidences (excluant les résidences d'agrément), la contiguïté des habitations (la dispersion de l'habitat constituant précisément un signal d'alerte face au risque d'aggravation du mitage et de l'étalement urbain), l'environnement immédiat (prise en contact de critères paysagers), l'équipement des constructions (garantie d'un impact écologique limité des aménagements).

Les juges évaluent le caractère continu de l'urbanisation par l'examen conjoint du caractère urbanisé des parcelles environnantes, et par celui des caractéristiques du projet envisagé. L'extrait de jugement suivant est représentatif du modèle de raisonnement régulièrement adopté : «Considérant que [...] le terrain ayant fait l'objet du certificat d'urbanisme attaqué n'est proche que d'une construction édifiée sur la parcelle 71 et de trois autres sur la parcelle 119 dont aucune ne lui est limitrophe; qu'il n'est pas situé ainsi dans une partie urbanisée de la commune; qu'il est éloigné de plus de 300 mètres des Marines de Sant-Ambroggio, lesquelles en tout état de cause, ne sauraient être regardées comme constituant une agglomération ou un village; que l'édification d'une maison ne saurait former à elle seule un hameau nouveau intégré à l'environnement; qu'ainsi, le préfet de la Haute-Corse [...] était tenu de délivrer un certificat d'urbanisme négatif $[. ..] »^{14}$.

14. Trib. Adm. Bastia, Commune de Lumio, 29 janvier 2004
Ces enjeux de définition expliquent que les argumentaires juridiques utilisés par les requérants (les «moyens de légalité ») renvoient aussi bien aux règles applicables à l'intérieur de certaines catégories d'espaces qu'à la détermination du contour de ces mêmes espaces. C'est ainsi qu'à côté des moyens de légalité traitant du principe général d'extension en continuité (présents dans $41 \%$ des affaires) se pose également la question de la qualification de certains espaces (Calmette, 2007). Tandis que les critères paysagers et géomorphologiques posés par la jurisprudence sont mobilisés dans le cas des « espaces proches du rivage » (8\% des affaires), les litiges concernant la caractérisation ou la délimitation des « espaces remarquables » (7\% des affaires) amènent les requérants à argumenter sur l'intérêt patrimonial des secteurs concernés, comme l'illustre bien un jugement de l'année 2007 concernant la commune d'Ajaccio : «(les requérants) soutiennent que le terrain d'assiette du projet fait partie d'un espace remarquable au sens de l'article L. 146-6 du Code de l'urbanisme; qu'en effet, d'une part, il se situe dans une partie naturelle du site inscrit du rivage nord du golf d'Ajaccio ; [...] que la parcelle en cause, d'une superficie de 5100 mètres carrés, ne supporte aucune construction et jouxte un ensemble naturel homogène comportant un couvert végétal dense et d'importants espaces boisés; qu'elle constitue, en raison de sa forte pente, une limite naturelle à l'urbanisation; que, d'autre part, le terrain s'insère dans une zone boisée nécessaire au maintien des équilibres biologiques et présentant un grand intérêt écologique [...] en raison de la présence de plantes rares et protégées, et de différentes espèces d'oiseaux et de reptiles et de batraciens également protégés [...] $\gg^{15}$

À côté de ces moyens de légalité mobilisant des dispositions propres à la loi

15. Trib. Adm. de Bastia, Commune d'Ajaccio, 14 septembre 2007. 
Figure 5. Moyens de légalité soulevés et catégories de demandeurs dans le contentieux administratif (analyse factorielle des correspondances)

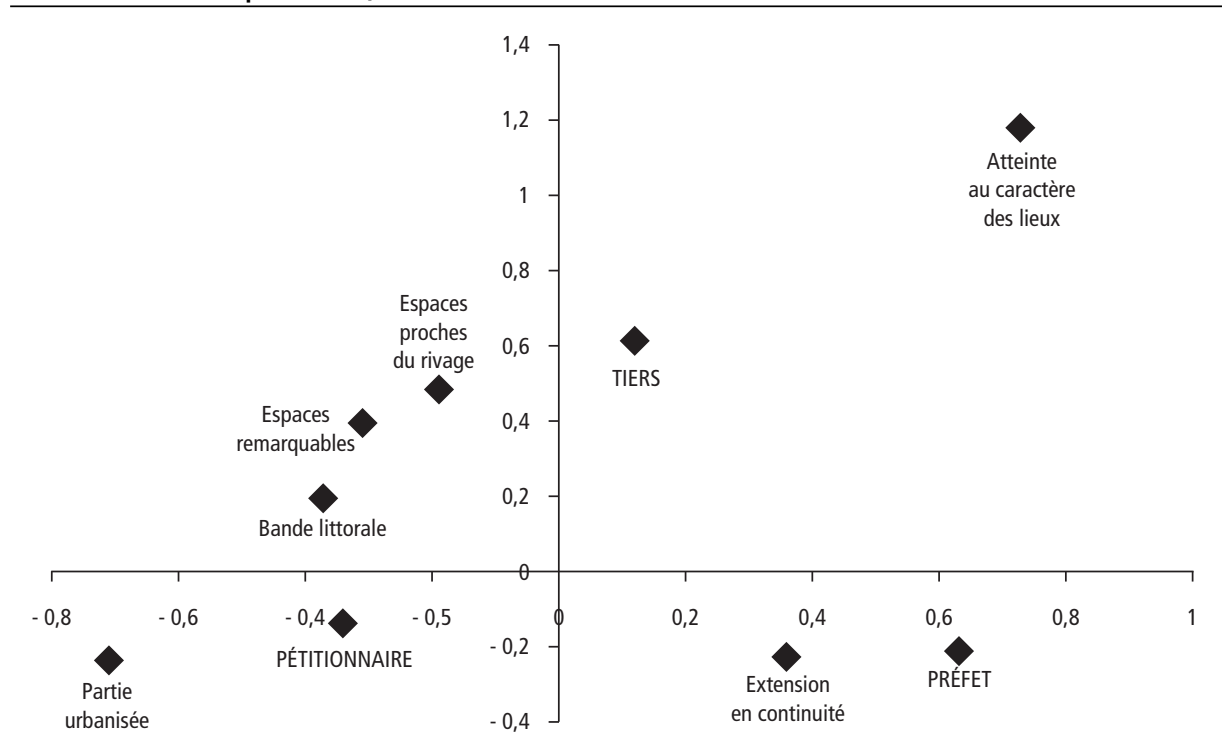

Source : Données de l'enquête

littoral, d'autres registres d'argumentation juridiques puisent dans les règles générales du droit de l'urbanisme en s'appuyant sur l'obligation générale imposée aux permis de construire, soit pour discuter du caractère urbanisé d'un secteur (en soulevant l'insuffisance ou non des équipements d'assainissement ou de voirie et les problèmes posés à la «salubrité et la sécurité publique ») ou pour étayer l'intérêt patrimonial d'un site (en arguant de l'atteinte ou au contraire du respect porté au « caractère des lieux »). En outre, en plus des règles législatives concurrentes à la loi littoral, ce sont les dispositions réglementaires locales édictées par les documents d'urbanisme qui sont également fréquemment mobilisées. Elles le sont généralement par des propriétaires désireux de s'appuyer sur le zonage ou le règlement communal à l'appui de leurs prétentions (un classement en zone constructible permet par exemple d'argumenter sur le caractère " d'espace urbanisé » d'un secteur). Les juges sont cependant peu ouverts à ce type d'argument, l'interprétation des dispositions de la loi littoral n'étant pas déterminée par les règles d'urbanisme édictées localement. À la diversité des moyens de légalité mobilisés se superpose la diversité des règles visées dans les mémoires des parties en fonction du type de demandeur partie prenante à la procédure : c'est en cela que l'on peut parler de registres d'argumentation attaché à un profil de requérant. Cette ouverture des registres d'argumentation n'aboutit pas forcément à renforcer les chances de succès de ces requérants. Les juges privilégient en effet la conformité à des principes généraux comme celui de l'extension en continuité et ne retiennent que rarement les moyens qui renvoient à la qualification d'espaces de nature spécifique (espaces remarquables ou proches du rivage) ou qui s'appuient sur des dispositions générales du droit de l'urbanisme. C'est d'ailleurs en suivant ce principe «d'économie » qui gouverne l'examen des requêtes que l'administration obtient un taux de réussite important devant le tribunal.

En effet, l'analyse factorielle représentée graphiquement dans la figure 3 permet de 
mettre en évidence les registres d'argumentation (représentés en lettres minuscules sur le graphique) associés aux différentes catégories de parties à l'origine des recours (représentées en lettres majuscules, soit : $1^{\circ}$ les «pétitionnaires », c'est-à-dire les personnes ayant sollicité le permis de construire ou toute autre autorisation d'urbanisme, $2^{\circ}$ les tiers attaquant les autorisations délivrées $3^{\circ}$ parmi lesquels figurent une catégorie particulière de «tiers », en l'occurrence le préfet). Alors que les registres d'argumentation proches des pétitionnaires et des tiers sont caractérisés par une relative variété et mobilisent les différentes catégories de la loi littoral (soit pour appuyer soit pour contester une extension des droits à construire), le représentant de l'État se contente généralement d'appuyer son argumentation sur le principe général d'extension en continuité de l'urbanisation.

\section{Conclusion}

Les dispositions de la loi littoral (reprises dans le code de l'urbanisme) assortissent le développement de l'urbanisation dans les communes concernées d'un certain nombre de conditions que doivent respecter les règles de planification (plans locaux d'urbanisme, cartes communales) et les autorisations administratives (permis de construire, certificats d'urbanisme). Pour ses contempteurs, la loi littoral conduit à un gel économique de vastes territoires, dont certains dotés d'un fort potentiel pour l'économie touristique, qui plus est dans une région marquée par un développement atone. Mais de fait, la réalité du dispositif juridique est complexe puisqu'il juxtapose des catégories d'espaces protégés et des catégories de règles de nature variable. En dehors de certaines situations ciblées (espaces remarquables, bande littorale), les dispositions protectrices jouent davantage le rôle d'aménagement de l'urbanisation qu'elles ne contiennent d'interdictions explicites. Le principe d'une régulation souple des protections, appelée de ses vœux par la majorité des élus du littoral dans le cadre du projet de plan d'aménagement régional, semble ainsi à bien des égards caractériser dès le départ le cœur de la législation d'urbanisme. À ceci s'ajoute le constat que le contexte de dynamisme du marché de la construction dans l'île n'a pas été durablement affecté par ces règles de protection, tandis que certains élus ont pu jusqu'à une période récente s'accommoder localement d'un contrôle limité des services de l'État sur les demandes d'autorisations.

Par ailleurs, les enjeux de protection des espaces littoraux doivent sans doute moins s'apprécier au regard de la position des juges en la matière (peu de jugements accèdent aux requêtes des propriétaires demandant l'annulation de refus d'autorisation) qu'en considération de la « clientèle des tribunaux » (Melot, 2009). Le nombre réduit de recours émanant de tiers, en particulier associatifs (en dépit d'une certaine réussite devant le prétoire) et le caractère paradoxal des déférés préfectoraux devant le tribunal administratif (à la fois nombreux et limités géographiquement) donne le sentiment que l'activisme judiciaire de ces « avocats naturels » de la protection du littoral repose sur des bases fragiles. Ce constat appelle une interrogation quant à l'avenir, sachant que la plupart des communes littorales se dotent progressivement de documents d'urbanisme, et que par voie de conséquence les services de l'État seront de moins en moins souvent en situation de se prononcer sur les demandes. La maîtrise de l'urbanisation pourra-t-elle être assurée par des élus qui n'opèrent qu'une sélection limitée sur les projets ? Ces enjeux, qui se posent de manière particulièrement aiguë dans le cas des zones sensibles sur le plan environnemental comme les espaces littoraux, illustrent sans doute d'une manière particulièrement aiguë la question de la capacité de pilotage, à un échelon aussi localisé que l'échelon communal, des compétences d'urbanisme. 


\section{RÉFÉRENCES BIBLIOGRAPHIQUES}

Barré M.-D., Aubusson de Cavarlay B., Zimolag M. (2006). Dynamique $d u$ contentieux administratif. Analyse statistique de la demande enregistrée par les tribunaux administratifs. Rapport pour la Mission de recherche Droit et justice.

Blanchard R. (1914). Les genres de vie en Corse et leur évolution. Recueil des travaux de l'Institut de géographie alpine, $\mathrm{n}^{\circ} 2$, p. 187-238.

Calderaro N. (1994). Construction du droit sur le littoral. Études foncières, $\mathrm{n}^{\circ} 74$, mars, p. 20-26.

Calmette J.-F. (2007). La rareté de l'espace littoral saisie par le juge administratif. Études foncières, $\mathrm{n}^{\circ} 126$, mars-avril, p. 20-23.

Celli A., Garcia T. (2002). L'adaptation à l'insularité du cadre juridique national relatif au littoral: les exemples corse et sarde. Revue juridique de l'environnement, $\mathrm{n}^{\circ} 3$, p. 393-412.

Daligaux J. (1996). Conflits sur l'habitat diffus dans le Var. Études foncières, $\mathrm{n}^{\circ} 71$, p. 11-15.

Daligaux J. (2003). Urbanisation et environnement sur les littoraux : une analyse spatiale. Rives méditerranéennes, $\mathrm{n}^{\circ} 15$, p. 11-20.

Galanter M. (1974). Why the "Haves" come out ahead: speculations on the limits of legal change. Law and society review, $\mathrm{n}^{\circ}$ 9, p. 95-160.

Kirat T. Melot R. (2006). Du réalisme dans l'analyse des conflits d'usage : les enseignements de l'étude du contentieux. Développement durable et territoires, http://developpementdurable.revues.org/d ocument2574.html

Kirat T., Torre A. (2007). Conflits d'usage et dynamiques spatiales : les antagonismes dans l'occupation des espaces ruraux et périurbains (II). Géographie, Économie, Société, p. 119-120.
Lascoumes P. (1995). Les arbitrages publics des intérêts légitimes en matière d'environnement. L'exemple des lois Montagne et Littoral. Revue française de science politique, vol. $45, \mathrm{n}^{\circ}$ 5, juin, p. 396-419.

Le Cornec E. (1997). La prise en compte de l'environnement par les règles locales d'urbanisme. Thèse pour le doctorat en droit public, Université Paris I.

Levratto N. (2001). Économie de la Corse. $Y$ a-t-il une vie après la rente? Éd. Albiana.

Maupertuis M.-A., Logossah K. (2007). La spécialisation touristique des petites économies insulaires en développement est-elle une voie de croissance durable ? Revue d'Économie Régionale et Urbaine, $\mathrm{n}^{\circ} 1$, p. 35-56.

Melot R. (2009). De la gestion des espaces au projet de territoire: les enjeux politiques d'un changement de paradigme juridique. L'Année sociologique, vol. 59, p. 177-199.

Paoli J.-C., Melot R., Fiori A. (2008). L'aménagement du territoire à l'épreuve de la décentralisation : conflits et concertation en Corse et Sardaigne. Pôle Sud: revue de science politique de l'Europe méditerranéenne, $\mathrm{n}^{\circ}$ 28-1, p. 143-165.

Paoli J-C. (2000). Politique globale et développement local : une lecture institutionnaliste du développement rural sarde. Economie rurale, $\mathrm{n}^{\circ} 263$, mai-juin, p. 35-47.

Struillou J.-F. (2004). L'application du droit pénal de l'urbanisme. Etat de droit et urbanisme, Cahier du GRIDAUH, $\mathrm{n}^{\circ} 11$.

Torre A., Caron A. (2005). Réflexions sur les dimensions négatives de la proximité : le cas des conflits d'usage et de voisinage. Economie et Institutions, 6 \& 7 , p. 183-220. 